\title{
THE SECOND POSTULATE OF RELATIVITY AND THE ELECTROMAGNETIC EMISSION THEORY OF LIGHT.
}

By O. M. Stewart

TNAMUCH as it appears that the results of the relativity principle are to be accepted or rejected as we accept or reject the two postulates, these postulates should receive very careful attention.

Einstein has stated the first postulate in a general form: The laws governing natural phenomena are independent of the motion of the system of coördinates used in observations on these phenomena, provided there is no acceleration of this system. In other words, one can never tell from any observations he may make what the absolute velocity of his origin of coördinates may be. The observed operation of any laws would be the same whether one is absolutely at rest or moving with a uniform linear motion. Two observers, one $A$, the other $B$, have relative motion. It is a matter of indifference whether we say $A$ is moving and $B$ at rest, or $B$ moving and $A$ at rest, since all observations made by $A$ can be duplicated by $B$. There thus arises an interesting symmetry which will be referred to later. This postulate has been accepted in mechanics since the days of Newton, and is confirmed in other branches of physics by all the experiments that man has been able to devise. Such for example are the experiments of Michelson and Morley, ${ }^{1}$ and Trouton and Noble. ${ }^{2}$

The second postulate, as stated by Einstein, is that light is always propagated in vacuo with the same velocity, this velocity being independent of any motion of the source. This postulate is based in part on experiment and in part on an assumption. The experiments of Michelson and Morley and others show that

${ }^{1}$ Am. Jour. Sci., 34, p. 333, I887.

${ }^{2}$ Phil. Trans. Roy. Soc., (A), 202, p. I65, I904. 
the time required for light to travel to a mirror and return is independent of any translatory motion of the apparatus as a whole. In other words, all experiments that have been made on the velocity of light show an agreement with the first postulate. The assumption is that the velocity of light is independent of the velocity of the source. We may then say that the results of the relativity principle are due to a generalization of a law of mechanics (the first postulate) and to the assumption that the velocity of light is independent of the velocity of the source. This assumpton has been generally accepted on account of our concept of the ether as a fixed medium filling all space. But, as has been shown by Einstein and others, the first postulate of relativity leads to the rejection of this concept of the ether. In fact, in all experiments where it has been possible to test this conflict between the stationary ether theory and the first postulate, the results have been in favor of the latter. The experiments already referred to, those of Michelson and Morley, and Trouton and Noble, are illustrations of this. Thus we have the principle of relativity destroying a concept which is used in one of its postulates.

It has been pointed out in many places that if we deny this assumption and admit that the velocity of light is a function of the velocity of the source, the complete explanation of such experiments as those of Michelson and Morley follows at once, and at the same time the strange conclusions of the relativity principle are destroyed. Such an hypothesis makes it necessary for us to modify our conception of the ether, but this we must do in either case. If we retain the old idea that the velocity of light is independent of the velocity of the source, we apparently must accept the relativity principle with its rejection of a fixed medium filling all space together with an entire revision of our concepts of the fundamental and derived units. At present it seems less revolutionary to assume that the velocity of light is a function of the velocity of the source. It is the purpose of this paper to attempt to clear up certain objections that have been raised to this view.

Sir J. J. Thomson ${ }^{1}$ has presented a theory of light which makes it possible for the velocity to be a function of the velocity of the

${ }^{1}$ Philosophical Magazine, I9, p. 30r, r9ro. 
source. This has been called by Kunz ${ }^{1}$ the electromagnetic emission theory. The electrons in the light source, according to this theory have attached to them tubes of force. When an electron is accelerated a transverse vibration is propagated out each of its tubes with a velocity equal to $c$, the velocity of light. These transverse vibrations or waves are what we call light waves. The "medium" through which light is propagated is in this theory these Faraday-Thomson tubes. If the source has a motion of translation these tubes also have the same velocity, provided this velocity is uniform. The "medium" always has the same uniform velocity as the source. Hence if $c$ is the velocity of light when the source is stationary, $c+v$ is the velocity when the source has a velocity whose component in the direction of the propagation of light is $v$. In other words, the velocity of light with respect to the source is always $c$.

Measurements of the Doppler Effect with a Grating.-It has been pointed out by both Tolman ${ }^{2}$ and Kunz ${ }^{3}$ that if the velocity of light changed in the manner just described, the length of a wave would not be changed by motion of the source. In the equation

$$
v=n \lambda
$$

both $v$ and $n$ are changed in such a way that $\lambda$ is not changed. Moreover the same wave-length would be emitted in all directions by a moving source. This has led Tolman to object to this theory on the ground that gratings which always measure wavelengths would not detect the Doppler effect from stars or the limb of the sun as we know they do. However the theory of the grating assumes a stationary medium. For example, in the elementary theory of the transmission grating it is assumed that when a wavelet "spreads" out from each one of the slits the center of the disturbance in the medium remains in the slit. This is readily seen if one obtains the resultant wave-front by Huyghens's construction, that is, by means of wavelets emanating from the openings. Whether or not we could detect this Doppler effect

${ }^{1}$ Amer. Jour. Sci., 30, p. 313, r910.

2 Phys. Rev., 3I, p. 26.

${ }^{3}$ Loc. cit. 
with a grating would depend on the nature of the "medium" that transmits these waves.

If light waves are the vibrations of electric tubes moving with the source as supposed in Thomson's theory, this medium in the case of a moving star would be drifting through the grating with a velocity equal to the velocity of the star. In this event the grating would show the Doppler effect. Moreover, it may be shown that the observed effect would depend merely on the relative motion of the star and the observer, that is, it would be the same whether the observer is in motion or whether it is the star that is moving. In other words, this obeys the first postulate of relativity. ${ }^{1}$ Thus if we change our conception of the medium as we must if we are to assume that the velocity of light depends on the velocity of the source, this difficulty disappears.

Tolman's Interference Experiment.-In the same paper Tolman describes an interesting experiment which leads him to raise another objection to the supposition that the velocity of light is changed by the motion of the source. The experiment consists in observing the interference fringes produced by Lloyd's mirror when first one limb of the sun and then the other is focused on the slit. Consider two beams of light both coming from the same limb of the sun one passing directly to the screen the other to the same point after being reflected at nearly grazing incidence. It is shown that if light came from the limb of the sun with a velocity $c \pm u$ where $\pm u$ is the velocity of the limb in the line of sight due to the rotation of the sun, and if light reflected by this mirror has its velocity changed to $c$, then the observed fringes should shift as one changed the source from one limb to the other. The negative result was regarded as proving that light from the limb of the sun did not have a velocity $c \pm u$. However, it was assumed that when light is reflected by a mirror at any angle the mirror becomes the source and the velocity of the reflected light depends only on the velocity of this mirror. The negative result may be regarded as merely

${ }^{1}$ The explanation of the Doppler effect in terms of the usually accepted theory does not agree with this postulate. If the source is moving and the observer fixed, the ether waves are said to be changed in length. On the other hand, if we consider the source as stationary and the observer moving there is no change in the wavelength. 
proving that this assumption is in general not correct. Much may be said in favor of the idea that when light is reflected, the mirror becomes a new source. But in view of the fact that we must modify our concept of the ether and the electromagnetic theory, this is clearly an assumption.

Certain considerations, to be explained later, lead to the law that the velocity of light reflected by a mirror will depend on the velocity of the image of the source, that the velocity after reflection will be equal to $c+v$, where $v$ is the component of the velocity of the image of the source in the direction of the reflected light. Applying this to the experiment of Tolman, we find that the image of the moving source, when formed by reflection at nearly grazing incidence in the mirror, has the same velocity as the source. Hence in this case the velocity of light would not be changed by the reflection and no shift of the fringes should be observed.

The Law of the Change of Velocity on Reflection.-This proposed reflection law is based on the electromagnetic emission theory of Thomson. One of the essential features of this theory is the reality given to the electric tubes of force. These are no longer to be geometrical fictions but to have a true objective existence. Since light is a disturbance propagated along these tubes, we may regard reflection as produced by a bending or reflection of these tubes. If we now assume no longitudinal compression of the tubes on reflection, the energy per unit volume in these tubes will not be altered by reflection from a perfect mirror. ${ }^{1}$ Further, as there will be no change in the longitudinal dimensions of the tubes there will be no change in the length of a wave on reflection. As a result of this assumption we have then two theorems: $(a)$ No change in the energy density, $(b)$ no change in the wave-length on reflection. Since this theory of Thomson shows that in the case of direct radiation, motion of the source produces no change in the energy density $^{2}$ or in the length of a wave these theorems are only parts of a

${ }^{1}$ Absence of longitudinal compression would mean inability to transmit longitudinal waves.

${ }^{2}$ It is conceivable that there may in certain cases be changes in the distribution of the tubes of force on account of the motion of the source, for example when moving through a magnetic field. In such a case there would be a change in the distribution of the intensity of radiation. This effect is neglected above. 
more general law which may be stated thus: Motion of a source, real or virtual, does not change either the energy density ${ }^{1}$ or the wave-length.

This leads to a symmetrical condition which is shown by the consideration of a source $A$ and a mirror $B$ having relative motion. Whether we consider $A$ at rest and $B$ moving, or $B$ at rest and $A$ moving, we have identically the same condition, that is, the same energy in the reflected beam. The same symmetry is shown in regard to the length of waves. The wave-length is not changed by motion of the source nor by reflection. In this respect we have complete agreement with the first postulate of relativity. Hence the difficulty pointed out in the footnote on p. 421 , which is found in the generally accepted theory, does not appear here.

We may by using either one of these theorems obtain the law of the velocity of reflected light. The constancy of the energy density will be used first. For brevity we shall take the case of normal incidence. Let the source have a velocity $u$, the mirror a velocity $v$, both velocities being in the same direction along the line joining the source and mirror. If the mirror were stationary it would receive energy at the rate of

$$
e A(c+u)
$$

per second, $e$ being the energy per unit volume in the incident beam near the mirror, $A$ the area of the beam, and $c+u$ the velocity of the incident light. But on account of the motion of the mirror not all of this energy is received, some being distributed over the space vacated by the mirror, the rate of loss being $e A v$. Hence the energy received per second is

$$
e A(c+u-v) \text {. }
$$

As the energy density of the reflected beam is to remain constant, the light must be carried away from the mirror at a velocity $c+u-v$ measured with respect to the mirror. When referred to the system of coördinates from which both $u$ and $v$ are measured the velocity of the reflected beam is

$$
c+u-2 v \text {. }
$$

${ }^{1}$ This energy density is what is later called the intrinsic energy density. 
It can readily be shown that the velocity of the image of the source seen in the mirror is

$$
2 v-u .
$$

Hence the velocity of the reflected light is

$$
c-(2 v-u),
$$

where $2 v-u$ is the velocity of the image, in this case in a direction opposite to that of the propagation of the reflected light. ${ }^{1}$

This can be extended to the more general case where the light falls on the mirror at any angle of incidence. To do this it must be noted that the tangential component of the mirror velocity will have no effect and that only the component of the normal velocity in the direction of the incident or reflected light need be considered. A general rule follows: The velocity of light is always $c+v$ where $v$ is the component in the direction of the propagation of light of the velocity of the source for direct light, of the image (virtual source) for reflected light.

The same result is obtained if we use the theorem that the wavelength is not changed by reflection. Since for any monochromatic beam of light $\lambda$ is always a constant, we have

$$
c^{\prime} / n^{\prime}=\lambda=\text { a constant, }
$$

where $c^{\prime}$ and $n^{\prime}$ are respectively the velocity and frequency relative to any observer. When light is reflected from a moving mirror,

\footnotetext{
${ }^{1}$ Some explanation of the use of the term energy density is necessary. As used above it may be defined as the energy absorbed from unit volume by a black body having the same velocity as the tubes of force, $i . e$., the same velocity as the source, real or virtual as the case may be. We may call this the intrinsic energy density. In general, the energy density is relative, depending on the relative velocity of the source and the observer. In the case where the observer has not the same velocity as the source the energy density he measures may be said to be the intrinsic density plus or minus a term depending on the work done against radiation pressure. It is a matter of indifference whether we say this work is done by the moving tubes or whether we say the source is stationary and the work is done by the moving observer. This is very similar to problems that arise in mechanics where the energy a body has is relative, depending on the choice of a system of coördinates. In the formulas given above, the changes in the energy due to the work done on account of radiation pressure are omitted, so that we are always dealing with this intrinsic energy. It may be noted that whenever the velocity of light is changed, work must be done and the relative energy density changed.
} 
both the velocity and the frequency may be changed and we will have

$$
c / n=c^{\prime} / n^{\prime}
$$

where $c$ and $n$ are the velocity and frequency before, and $c^{\prime}$ and $n^{\prime}$ the same quantities after reflection. Since $n^{\prime} / n$ may be observed, this may be written in the form

$$
n^{\prime} / n=c^{\prime} / c
$$

If we take the case of a source stationary with respect to the observer and a mirror moving with a velocity $v$ away from the source, a computation of the Doppler effect gives us

$$
\frac{n^{\prime}}{n}=\frac{c-2 v}{c}
$$

But this must equal the ratio $c^{\prime} / c$.

Hence

$$
c^{\prime}=c-2 v .
$$

Since the image has a velocity in this case equal to $-2 v$ we find the same result as before.

The Doppler Effect.-As just shown the change in frequency due to motion either of the source or of the mirror is given by

$$
n^{\prime} / n=c^{\prime} / c
$$

In applying this law $n^{\prime}$ may be regarded as the observed frequency and $n$ the frequency when there is no relative motion with respect to the observer. The velocities $c^{\prime}$ and $c$ must then be the velocities relative to this observer in the two cases. The law is independent of absolute motion. On the other hand in computing the change in frequency produced by the Doppler effect on the basis of a fixed medium, it is well known that the result is not independent of a choice of coördinates. For example, take the case of a source and an observer having relative motion. If we assume the source moving with a velocity $v$ toward the stationary observer, the ratio of the frequencies is

$$
\frac{n^{\prime}}{n}=\frac{c}{c-v}
$$


While, if we assume that the observer is moving and the source fixed we have

$$
\frac{n^{\prime}}{n}=\frac{c+v}{c} .
$$

That these two forms are different is in direct conflict with the first postulate of relativity.

In the electromagnetic emission theory, where motion produces no change in the wave-length, no such difference appears. No matter whether we regard the source moving or the observer we have for the case just cited

$$
\frac{n^{\prime}}{n}=\frac{c+v}{c}
$$

Kunz $z^{1}$ has suggested that simultaneous measurements be made on the velocity of Kanal rays and the Doppler effect produced by their motion to distinguish between this last formula and the one predicted by the relativity principle which is still different from any of the above. This experiment could also be used to distinguish between the above conflicting laws, provided the spectroscopic work be carried to a very high degree of accuracy.

Two Applications of the Electromagnetic Emission Theory.-Two examples of the use of the electromagnetic emission theory will be given. The first cannot be explained by the generally accepted theory without bringing in some strange hypothesis. The second when interpreted by the same theory leads to a violation of the first postulate of relativity. Experiments show that the time required by light to go to a distant mirror and return is independent of any velocity given to the whole apparatus. Let $A$ and $B$ be the source and mirror respectively, and $v$ their common velocity in the direction $A B$. According to this theory the light will leave $A$ with a velocity $c+v$ and since the image of $A$ in the mirror $B$ has the same velocity as both $A$ and $B$, the return velocity will be $c-v$. The time $t_{1}$ required for light to go from $A$ to $B$ is

$$
t_{1}=\frac{A B+v t_{1}}{c+v}=\frac{A B}{c} .
$$

1 Amer. Jour. Sci., 30, p. 319, I910. 
The time of the return will be

$$
t_{2}=\frac{A B-v t_{2}}{c-v}=\frac{A B}{c} .
$$

Or the total time is $2 A B / c$, a quantity independent of the vector $v$. The Michelson-Morley experiment is a very accurate method for comparing the time required to make the round trip when the line $A B$ lies in the direction of the motion and when at right angles to it. The above expression is in complete agreement with their results in that it shows that the time is independent of any motion of $A B$.

The second case is that of two observers, who, while they have relative motion with respect to each other, exchange light signals. Let them agree to send at a predetermined instant a light flash to the other station, where it is to be reflected directly back. At first we may assume that $A$ is at rest and that $B$ has a velocity $v$ directed away from $A$. The time required for light to go from $A$ to $B$ is

$$
t_{1}=\frac{A B+t_{1} v}{c}=\frac{A B}{c-v},
$$

where $A B$ is the distance between $A$ and $B$ at the instant the flash starts. The time of the return will be

$$
t_{2}=\frac{A B+t_{1} v}{c-2 v}=\frac{c}{(c-v)(c-2 v)} A B,
$$

the velocity of the light reflected from $B$ being $c-2 v$, as the image of $A$ has a velocity 2v. The time for the light flash starting at $B$ to go to $A$ will be

$$
t_{1}=\frac{A B}{c-v},
$$

the velocity now being $c-v$. The return time from $A$ to $B$ will be

$$
t_{2}=\frac{A B+\left(t_{1}+t_{2}\right) v}{c-v},
$$

the image of $B$ in $A$ 's mirror moving with a velocity $-v$. This time reduces to

$$
t_{2}=\frac{A B+t_{1} v}{c-2 v}=\frac{c}{(c-v)(c-2 v)} A B .
$$


Hence both signals, starting at the same time, one from $A$, the other from a moving observer $B$, would reach the other station at the same instant and return at the same instant. There would be no discrepancy in their ideas about the simultaneity of two events and no trouble in their time standards such as arise when the details of this experiment are computed on the assumptions of the relativity principle. The same results are obtained whether we regard $A$ as stationary and $B$ moving, or $A$ in motion and $B$ stationary, that is, the result is independent of all conception of absolute motion. It is therefore in complete agreement with the first postulate of the relativity principle.

We thus find that if we chose a suitable "medium," one that will account for light having a velocity which is dependent on the velocity of the source, we can explain the objections raised by Tolman. The electromagnetic emission theory seems so far to agree with observation in those cases where the present theory fails. Further, it is in accord with the first postulate of relativity and leads if accepted to the overthrow of the second postulate.

UNIVERSITY OF MisSOURI, December, rgro. 